\title{
Avaliação da qualidade dos vídeos sobre câncer de mama mais visualizados no YouTube: relevância para promoção da saúde da mulher
}

\author{
Quality assessment of the most watched breast cancer videos on YouTube: \\ relevance to improve women's health
}

\section{Evaluación de la calidad de los videos sobre el cáncer de mama más vizualizados en YouTube: relevancia para promover la salud de la mujer}

Larissa Silveira Carvalho Villa ${ }^{1, a}$

larissascvilla@hotmail.com | https://orcid.org/0000-0002-9218-4497

Alex Del Cerro Mello ${ }^{2, b}$

alexdelcerr25@gmail.com | https://orcid.org/o0oo-0002-9947-2942

José Vicente Gonçalves ${ }^{3, c}$

jgvz@uol.com | https://orcid.org/oooo-0003-4970-0481

Tânia Maria Gomes da Silva ${ }^{1,3, d}$

tania.gomes@unicesumar.edu.br | https://orcid.org/oooo-0002-5495-9968

Marcelo Picinin Bernuci ${ }^{i, 3, e}$

marcelo.bernuci@unicesumar.edu.br| https://orcid.org/oooo-0003-2201-5978

\footnotetext{
${ }^{1}$ Universidade Cesumar, Programa de Pós-graduação em Promoção da Saúde. Maringá, PR, Brasil.

2 Universidade Cesumar, Curso de Medicina. Maringá, PR, Brasil.

${ }^{3}$ Universidade Cesumar, Instituto Cesumar de Ciência Tecnologia e Inovação. Maringá, PR, Brasil.

a Mestrado em Promoção da Saúde pela Universidade Cesumar.

b Graduando em Medicina pela Universidade Cesumar.

c Mestrado em Estatística pela Universidade Federal de Brasília.

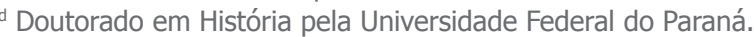

e Doutorado em Fisiologia pela Universidade de São Paulo.
}

\section{RESUMO}

O câncer de mama é uma das principais enfermidades que acomete a saúde das mulheres no mundo e as estratégias destinadas à melhoria do acesso das mulheres às informações sobre prevenção são fundamentais. O objetivo do estudo apresentado neste artigo foi avaliar a qualidade de vídeos relacionados ao tema do câncer de mama. A busca dos vídeos ocorreu a partir da palavra-chave 'câncer de mama' na plataforma YouTube. Os dados referentes às informações de cunho técnico foram obtidos por meio da análise das informações contidas na descrição dos vídeos e, posteriormente, foi realizada sua análise de conteúdo. Para serem analisados, foram extraídos os 200 primeiros vídeos mais vistos. A maioria (72\%) e aqueles com intuito de divulgar conteúdo informativo ao leigo (73\%) e com foco na prevenção secundária (71,5\%) são considerados ruins ou péssimos (53\%). Conclui-se, que o YouTube se apresenta como uma ferramenta limitada a ser utilizada no processo de educação de mulheres visando ao controle do câncer de mama.

Palavras-chave: Medicina preventiva; Redes sociais online; Comunicação em saúde; Internet; Doença crônica não-transmissível. 


\section{ABSTRACT}

Breast cancer is one of the main diseases that affect the health of women in the world and strategies aimed at improving women's access to information on prevention are fundamental. The objective of the study presented in this article was to assess the quality of some videos related to breast cancer. The videos were searched using the keyword 'breast cancer' on the YouTube platform. The data referring to the technical information was obtained through the analysis of the information contained in the description of the videos and subsequently their content analysis was carried out. The first 200 most viewed videos were extracted for analysis. The majority (72\%) and those aiming to disseminate informative content to laypeople (73\%) and focusing on secondary prevention (71.5\%) are considered bad or very bad (53\%). It was concluded that YouTube is a limited tool to be used in the process of educating women about the control of breast cancer.

Keywords: Preventive medicine; Online social networks; Health communication; Internet; Chronic noncommunicable disease.

\section{RESUMEN}

El cáncer de mama es una de las principales enfermedades que afectan la salud de las mujeres en el mundo y las estrategias destinadas a mejorar el acceso de las mujeres a la información sobre prevención son fundamentales. El objetivo del estudio presentado en este artículo fue evaluar la calidad de videos relacionados con el tema del cáncer de mama. Los videos fueran buscados utilizando la palabra clave 'cáncer de mama' en la plataforma de YouTube. Los datos referentes a la información técnica se obtuvieron mediante el análisis de la información contenida en la descripción de los videos y posteriormente se realizó su análisis de contenido. Fueron extraídos los primeros 200 videos más vistos para hacer su análisis. La mayoría (72\%) y aquellos que tienen la finalidad de difundir contenidos informativos al lego en la materia (73\%) y enfocon la prevención secundaria $(71,5 \%)$ son considerados de poca calidade o pésimos (53\%). Se concluye que YouTube se revela una herramienta limitada para ser utilizada en el proceso de educación de las mujeres destinada al control del cáncer de mama.

Palabras clave: Medicina preventiva; Redes sociales online; Comunicación acerca de la salud; Internet; Enfermedad crónica no transmisible.

Contribuição dos autores:

Concepção e desenho do estudo: Larissa Silveira Carvalho Villa, Marcelo Picinin Bernuci.

Aquisição, análise ou interpretação dos dados: Larissa Silveira Carvalho Villa, Alex Del Cerro Mello, José Vicente Gonçalves. Redação do manuscrito: Larissa Silveira Carvalho Villa, Alex Del Cerro Mello, José Vicente Gonçalves.

Revisão crítica do conteúdo intelectual: Tânia Maria Gomes da Silva, Marcelo Picinin Bernuci.

Declaração de conflito de interesses: não há.

Fontes de financiamento: O presente trabalho foi realizado com apoio da Coordenação de Aperfeiçoamento de Pessoal de Nível Superior - Brasil (CAPES) - Código de Financiamento 001 e do Instituto Cesumar de Ciência, Tecnologia e Inovação - ICETI.

Considerações éticas: não há.

Agradecimentos/Contribuições adicionais: Ao Instituto Cesumar de Ciência, Tecnologia e Inovação (ICETI).

Histórico do artigo: submetido: 17 mar. 2021 | aceito: 14 jun. 2021 | publicado: 31 ago. 2021.

Apresentação anterior: não há.

Licença CC BY-NC atribuição não comercial. Com essa licença é permitido acessar, baixar (download), copiar, imprimir, compartilhar, reutilizar e distribuir os artigos, desde que para uso não comercial e com a citação da fonte, conferindo os devidos créditos de autoria e menção à Reciis. Nesses casos, nenhuma permissão é necessária por parte dos autores ou dos editores. 
Reciis - Revista Eletrônica de Comunicação, Informação \& Inovação em Saúde, Rio de Janeiro, v. 15, n. 3, p. 648-664, jul.-set. 2021 [www.reciis.icict.fiocruz.br] e-ISSN 1981-6278

\section{INTRODUÇÃO}

O câncer tem se tornado, nos últimos tempos, um problema de saúde pública mundial, que afeta especialmente os países subdesenvolvidos pelas dificuldades na implementação de políticas públicas de prevenção e detecção precoce (ORGANIZAÇÃO PAN-AMERICANA DE SAÚDE, 2020). No que tange à saúde da mulher, o câncer de mama é um dos mais prevalentes, e apenas no ano de 2018 mais de 2 milhões de novos casos foram detectados (GLOBOCAN, 2020). Em 2020, estimaram-se 2,3 milhões de novos casos de câncer de mama, representando 11,7\% do total de câncer na população mundial, configurando-se uma das principais doenças que acomete a saúde das mulheres no mundo (GLOBOCAN, 2020). No Brasil, o cenário ainda é pior; segundo dados do Instituto Nacional do Câncer (INCA), o câncer de mama é o segundo tipo de câncer que mais acomete as brasileiras (INCA, 2020) e a principal causa de morte dessa população (GLOBOCAN, 2020).

Embora as ações governamentais voltadas para o controle dessa malignidade tenham sido intensificadas desde 2010, até o momento, os avanços na redução das taxas de mortalidade ainda são incipientes, devido, principalmente, à demora no diagnóstico (BRASIL, 2013). Nos últimos anos, o governo brasileiro, por meio da implementação de políticas públicas para o enfrentamento das doenças crônicas não transmissíveis, tem adotado diversas estratégias de conscientização da população feminina quanto à importância do diagnóstico precoce do câncer de mama (FIGUEIREDO et al., 2017; DOS-SANTOS-SILVA, 2018). Evidências de que o diagnóstico tardio do câncer de mama está relacionado com a baixa consciência e o desconhecimento sobre o processo saúde-doença pelas mulheres (HERR et al., 2013) apontam para necessidade de subsidiar informações sobre a prevenção deste tipo de câncer para a população.

Mesmo que se reconheça os esforços governamentais para implementar estratégias de educação em saúde, não se pode ignorar os efeitos negativos da propagação de notícias falsas e/ou conteúdos informativos equivocados, por indivíduos, ou associações não especializadas na área da saúde, que se utilizam de diferentes veículos de comunicação para influenciar a população (AMORIM et al., 2008; PINHEIRO et al., 2019). Não se pode, também, descartar a influência de outros fatores, classicamente descritos como facilitadores de má gestão do autocuidado em saúde, como baixa literacia em saúde, falta de possibilidades de acesso ao cuidado ou até mesmo pouca oportunidade de oferta de serviços de saúde de qualidade, os quais mantêm estreita relação com educação, status social, sexo, idade, área de habitação e outras condições de vida (TENGLAND, 2016). Neste sentido, as estratégias de educação em saúde, especialmente aquelas ditas 'empoderadoras', que se correlacionam ao processo de emancipação dos sujeitos (VALE, 2018), podem facilitar a quebra das barreiras de desigualdade e impulsionar a busca da diminuição das iniquidades, favorecendo, portanto, a melhoria do autocuidado e, no caso do câncer de mama, uma maior adesão aos programas de prevenção.

Ademais, nas últimas décadas, a preocupação com a segurança do paciente tornou-se assunto prioritário na área da saúde (FROTA et al., 2013; SALVADOR, 2017), e embora ainda não haja uma regulamentação para o controle das informações divulgadas nos diferentes veículos de comunicação, espera-se que os profissionais da área da saúde, associações governamentais ou não governamentais envolvidas com a saúde, ocupem cada vez mais espaço nesse cenário, e passem a atuar com mais solidez e comprometimento no processo de capacitação em saúde, tanto no âmbito individual quanto coletivo. Como a educação em saúde não se restringe à propagação de informações voltadas para a saúde, mas sim à promoção da motivação, das habilidades e da confiança necessárias para se adotar medidas que melhorem a saúde (WHO, 2013), as estratégias de promoção da saúde, baseadas no 'empoderamento', se apresentam como uma nova alternativa para resolução de diversos problemas de saúde pública (SADEGHI; HESHMATI, 2019), em especial, relativos ao câncer de mama, cuja sobrevida das mulheres aumenta significativamente quando o diagnóstico é realizado precocemente. Tendo em vista o uso crescente de plataformas na $w e b$ para interagir, 
gerar, acessar e disseminar informações em saúde, a preocupação com a qualidade e, principalmente, com a veracidade das informações disponibilizadas têm assumido relevância ainda maior para a saúde pública (FROSSARD; DIAS, 2016; BENEDICTIS et al., 2019).

Inúmeros estudos têm sido desenvolvidos a fim de investigar as implicações dessa nova 'forma' de educação no contexto da saúde, em especial sobre a disseminação das fake news por meio das redes sociais online (RSO). Até o momento, os dados gerados são contraditórios e, mesmo se reconhecendo os ganhos desse novo método na capacitação dos indivíduos sobre diversos tópicos de saúde, os efeitos da disseminação de conteúdos equivocados, ou tendenciosos, que normalmente perpetuam ideias de teorias conspirativas, contam a desfavor de sua aplicabilidade na saúde pública (MORETZSOHN, 2017; SILVA; SILVA JUNIOR, 2019). Dentre estas RSO, destaca-se a plataforma de compartilhamento de vídeos YouTube como uma das principais ferramentas utilizadas no processo de educação em saúde na contemporaneidade (KELLY et al., 2016; CURRAN et al., 2017; PANDER; PINILLA; DIMITRIADIS; FISCHER, 2019; FRANÇA; RABELLO; MAGNAGO, 2019). O YouTube é a maior plataforma de compartilhamento de vídeos do mundo e, atualmente, há registros de que mais de 70\% dos indivíduos adultos utilizam essa plataforma para criar ou disseminar conteúdos informativos diversos (LANGFORD; LOEB, 2019). Com mais de 100 milhões de vídeos em sua plataforma (CHOU; HUNT; FOLKERS; AUGUSTSON, 2017), o YouTube pode se apresentar como uma valiosa ferramenta de educação em saúde, desde que o conteúdo das informações seja de fato de qualidade. Como qualquer pessoa pode publicar um vídeo sobre saúde no YouTube, independentemente de sua formação, qualificação médica, profissão ou intenção, não é difícil reconhecer sua fragilidade como ferramenta de educação em saúde.

Evidências da heterogeneidade da qualidade dos conteúdos dos vídeos disponíveis sobre tópicos de saúde no YouTube, desde conteúdo de alta qualidade até propaganda de vendas ou golpes pseudocientíficos (CHOU; HUNT; FOLKERS; AUGUSTSON, 2017; BACKINGER et al., 2011; DAWSON et al., 2011; SYEDABDUL et al., 2013) reforçam a necessidade de cuidado com a adoção dessa ferramenta como estratégia de educação em saúde. Esforços têm sido realizados a fim de padronizar a publicação de vídeos com conteúdos relacionados à saúde no YouTube como, por exemplo, o Centro de Controle e Prevenção de Doenças (CDC - Centers for Disease Control and Prevention) nos Estados Unidos, que desenvolveu uma diretriz específica para publicação no YouTube e em outros sites de vídeo online. Mesmo com esses esforços, os estudos atuais apontam sérias limitações na qualidade dos vídeos sobre questões de saúde no YouTube (SELVI et al., 2019; SOUZA et al., 2019; ALBARADO et al., 2019; LIMA et al., 2019; MOTA et al., 2019), e reforçam a necessidade de mais iniciativas para tentar mitigar os efeitos negativos dessa fonte disseminadora de conteúdo na $w e b$.

No que tange ao tema do câncer de mama, poucos estudos foram realizados com foco no conteúdo dos vídeos do YouTube. Até o momento, os objetos de estudo foram vídeos específicos sobre mastectomia (HILLYER et al., 2019), autoexame e mamografia (BASCH et al., 2015), sem um foco específico na relevância de conteúdos de cunho informativo sobre a prevenção desse tipo de câncer. Diante, portanto, da importância da divulgação de informações de qualidade sobre a prevenção do câncer de mama para a população e da ausência de estudos dedicados à análise da qualidade das informações sobre o câncer de mama nos vídeos do YouTube, investigamos no presente estudo a potencialidade do uso da plataforma como estratégias de educação em saúde no âmbito da prevenção do câncer de mama.

\section{METODOLOGIA}

Trata-se de um estudo descritivo da análise de conteúdo de vídeos da plataforma YouTube sobre o câncer de mama, coletados nos meses de outubro e novembro de 2020. O estudo não foi registrado nem avaliado pelo sistema CEP/Conep conforme Inciso III, Parágrafo Único, do Artigo $1^{\circ}$ da Resolução 512 de 2016 do 
Conselho Nacional de Saúde, pois utilizou informações de acesso público, como prescrito nos termos da Lei $\mathrm{n}^{0}$ 12.527, de 18 de novembro de 2011. Todas as informações apresentadas tiveram suas identificações ocultas, de forma a garantir o anonimato das publicações. Todo o delineamento experimental, coleta e análise dos dados foi desenvolvido seguindo métodos semelhantes a estudos anteriores que avaliaram vídeos do YouTube.

A seleção dos vídeos no YouTube ocorreu a partir da utilização da ferramenta de busca, utilizando a palavra-chave 'câncer de mama'. Como normalmente as pessoas costumam clicar nas primeiras páginas dos resultados da pesquisa, para selecionar os vídeos mais vistos e cada página contém aproximadamente 20 vídeos, foram extraídos para análise os 200 primeiros mais vistos, determinados por classificações de acordo com a contagem de visualizações, segundo o filtro da própria plataforma. Esses vídeos foram salvos em uma lista de reprodução para facilitar a revisão, uma vez que os resultados da pesquisa no YouTube podem mudar diariamente. O download dos vídeos foi realizado com auxílio do software ATube Catcher para análise offline, entre os dias 11 de outubro e 14 de novembro de 2020.

Todos os vídeos $(\mathrm{N}=200)$ foram analisados por dois avaliadores independentes. Ambos desconheciam a avaliação um do outro e, no caso de falta de consenso, a decisão final foi tomada por um terceiro membro da equipe especialista. A fim de avaliar apenas os vídeos com potencial de uso para educação em saúde, foram excluídos aqueles com duração superior a 15 minutos $(\mathrm{N}=36)$; inferior a um minuto $(\mathrm{N}=03)$; e, aqueles que não abordavam conteúdo pertinente ao tema do câncer de mama $(\mathrm{N}=$ o3). Desse modo, 158 vídeos foram incluídos no estudo.

Os dois avaliadores independentes assistiram a todos os vídeos selecionados $(\mathrm{N}=158)$ e os transcreveram integralmente, considerando suas características técnicas e de conteúdo. Os dados referentes às informações de cunho técnico foram obtidos por meio da análise das informações contidas na descrição dada pelo YouTube para cada vídeo. As características técnicas analisadas foram: duração; número de visualizações diárias; número de curtidas e não curtidas; quantidade de comentários; a identidade da conta que publicou o vídeo e o objetivo do vídeo.

Quanto à identidade da conta que publicou, os vídeos foram categorizados segundo a autodeclaração do perfil de conta: a) profissionais da saúde (médicos, enfermeiros, fisioterapeutas, fonoaudiólogos e acadêmicos de cursos em saúde); b) profissionais de outras áreas (mídia-propaganda, canal de notícias, programa de entrevistas); c) postados por ambos os profissionais (saúde e outra área); d) pessoa comum (paciente e demais pessoas que não são da área da saúde; e e) vídeos postados por sociedades públicas.

Quanto ao objetivo do conteúdo da postagem, os vídeos foram categorizados em: a) educação profissional; b) informação ao leigo; c) experiência pessoal; d) terapias alternativas; e e) outros (vídeos sem objetivo definido).

A análise das informações contidas no conteúdo dos vídeos, como ferramenta de educação em saúde direcionada ao controle do câncer de mama, foi realizada a partir da categorização dos vídeos, segundo abordagens definidas pelo Ministério da Saúde no controle dessa malignidade (MIGOWSKI et al, 2018). As abordagens analisadas foram:

a) Definição do câncer de mama: doença amplamente prevenida; uma das principais causas de morte por câncer nas mulheres; causada pela multiplicação desordenada de células da mama. Esse processo gera células anormais que se multiplicam, formando um tumor.

b) Epidemiologia: o câncer de mama é o tipo mais comum entre as mulheres, no mundo e no Brasil, e a sua maior incidência ocorre após os 35 anos, sendo ainda mais frequente após os 50 anos. São 1,38 milhão de novos casos e 458 mil mortes pela doença por ano. 
c) Fatores de risco: o câncer de mama não tem uma causa única. Diversos fatores estão relacionados ao aumento do risco de desenvolver a doença, tais como: idade, fatores endócrinos/história reprodutiva, fatores comportamentais/ambientais e fatores genéticos/hereditários.

d) Sinais e sintomas: o câncer de mama pode ser percebido pela mulher, na maioria dos casos, por meio dos seguintes sinais e sintomas: nódulo (caroço), fixo e geralmente indolor, é a principal manifestação da doença, estando presente em cerca de 90\% dos casos quando o câncer é percebido pela própria mulher; pele da mama avermelhada, retraída ou parecida com casca de laranja; alterações no bico do peito (mamilo); pequenos nódulos nas axilas ou no pescoço; e, saída espontânea de líquido anormal pelos mamilos.

e) Prevenção primária: a prevenção primária do câncer de mama está relacionada ao controle dos fatores de risco conhecidos e à promoção de práticas e comportamentos considerados protetores. Os fatores hereditários e os associados ao ciclo reprodutivo da mulher não são, em sua maioria, modificáveis; porém, fatores como excesso de peso corporal, inatividade física, consumo de álcool e terapia de reposição hormonal, são, em princípio, passíveis de mudança.

f) Prevenção secundária: as estratégias para a detecção precoce do câncer de mama são o diagnóstico precoce (abordagem de pessoas com sinais e/ou sintomas iniciais da doença) e o rastreamento (aplicação de teste ou exame numa população sem sinais e sintomas sugestivos de câncer de mama, com o objetivo de identificar alterações sugestivas de câncer e, a partir disso, encaminhar as mulheres com resultados anormais para investigação diagnóstica).

g) Prevenção terciária: tratamento local: cirurgia e radioterapia (além de reconstrução mamária); tratamento sistêmico: quimioterapia, hormonioterapia e terapia biológica.

A avaliação da qualidade do conteúdo dos vídeos foi realizada por meio da análise de acurácia. Segundo Eysenbach $e$ Köhler (2002), a acurácia como um critério de avaliação da qualidade da informação na $w e b$ é definida pelo grau de concordância entre a informação oferecida e a melhor evidência geralmente aceita pela prática médica. Dessa forma, foi realizada a comparação entre o conteúdo das informações contidas nos vídeos com aquelas publicadas pelo Ministério da Saúde (INCA, 2020). Para esta análise, foi construído um instrumento de análise baseado nas recomendações do Ministério da Saúde. Os dados foram preenchidos pelos avaliadores, por meio de um formulário online (Google Forms). Nesse formulário, sete tópicos foram avaliados quanto ao grau de concordância com o proposto pelo Ministério da Saúde (INCA, 2020). Os tópicos avaliados foram: 1) definição do câncer de mama; 2) epidemiologia; 3) fatores de risco; 4) sinais e sintomas; 5) prevenção primária; 6) prevenção secundária; e, 7) prevenção terciária. Cada avaliador utilizou uma escala tipo Likert (1 a 5) para avaliar cada abordagem, seguindo a correspondência:

1. As informações presentes no vídeo são totalmente erradas;

2. As informações presentes no vídeo são parcialmente erradas;

3. O vídeo não abrange nenhum dos tópicos selecionados;

4. As informações presentes no vídeo são concordantes e parcialmente abrangentes; e

5. As informações presentes no vídeo são totalmente concordantes e totalmente abrangentes.

Desse modo, todo vídeo avaliado recebeu uma qualificação quanto à acurácia das informações contidas, como: totalmente erradas, parcialmente erradas, não abrange os tópicos, parcialmente certas. Para avaliar a qualidade dos vídeos, com base na acurácia das informações, foi utilizada a correspondência entre as notas obtidas na escala de Likert e a denominação de qualidade, como: péssimo, ruim, regular, bom, ótimo. A classificação seguiu, portanto, a seguinte ordem: nota 01: péssimo; nota 02: ruim; nota 03: regular; nota 
04: bom; e nota 05: ótimo. Desse modo, cada vídeo avaliado recebeu um escore, que foi utilizado para qualificá-lo como péssimo, ruim, regular, bom ou ótimo.

Os dados são apresentados como média aritmética aparada, desvio padrão e mediana para dados numéricos, bem como frequências absoluta e relativa para dados categóricos. O teste de normalidade Shapiro-Wilk foi utilizado para a análise da distribuição de normalidade, na qual se constatou a normalidade dos dados, sendo, portanto, aplicado o método de Sturges de modo a corrigir o número de classes (Sturges: $\mathrm{k}=1+3,22 \log \mathrm{N}$ ) para as análises. A coerência das análises realizadas pelos dois avaliadores foi determinada por meio da análise de Kappa ponderado. Como os resultados de todas as avaliações apresentaram valores de Kappa do tipo moderado ( $\mathrm{p}<\mathrm{0,05}$ ), considerou-se pouca divergência entre os avaliadores, e os dados considerados no estudo se referem, portanto, à análise de um único avaliador. Todas as análises foram realizadas utilizando o programa estatístico IBM-SPSS V.27.

\section{RESULTADOS}

Os dados da distribuição de frequências das características técnicas dos vídeos estão apresentados na Tabela 1. No total, 158 vídeos foram analisados, com média aritmética aparada de duração igual a 4,58 \pm 3,28 minutos, com mediana de 3,66 minutos. A maioria dos vídeos (72,2\%) teve duração inferior a 6 minutos, e apenas 27,8\% tiveram duração superior a isso. O intervalo de tempo entre a publicação do vídeo e o download para análise apresentou média aritmética aparada de 2,63 \pm 2,11 anos com mediana de 2,15 anos. A maioria (89,2\%) foi publicada em menos de cinco anos da análise e 10,8\% demandaram mais do que esse período. O número total de visualizações dos vídeos foi de 4.494.343, com média aritmética aparada de visualização de $15.715 \pm 69.856$ por vídeo e mediana de 6.677 . A maioria dos vídeos $(73,4 \%)$ apresentou número de visualizações inferior a 18.000 , e 26,6\% superaram esse montante.

Quanto à identidade da conta que postou o vídeo, a maioria (67,7\%) foi de profissionais da área de saúde, e o restante $(32,3 \%)$ foi postado por profissionais de outra área ou por uma combinação de profissionais da área da saúde e de usuários que se declararam independentes, como pacientes e outros). Observou-se, que 115.621 pessoas deram os "likes" nos vídeos, com uma média aritmética aparada e desvio padrão de $732 \pm 1.891$ aprovações por vídeo. A maioria dos vídeos (53,2\%) recebeu menos de 200 aprovações. Já o total de reprovação foi de 2.454, resultando em uma média aritmética aparada de 7,93 \pm 43,95 reprovações por vídeo e mediana de 3 . A maioria dos vídeos $(59,5 \%)$ recebeu menos de quatro aprovações. A média aritmética aparada do número de comentários foi de 16,51 \pm 99,04 comentários por vídeo e mediana de 5 . A maioria $(61,4 \%)$ dos vídeos recebeu menos de 26 comentários. Quanto ao objetivo dos vídeos, a maioria $(72,8 \%)$ apresentou conteúdo informativo sobre algum aspecto do câncer de mama (seja para informar ao leigo ou ao profissional de saúde). Informações oriundas de relatos pessoais também foram preponderantes $(24,1 \%)$, por meio de vídeos que objetivaram o compartilhamento de experiências. 
Tabela 1 - Distribuição de frequências dos dados relativos à caracterização técnica dos vídeos

(continua)

\begin{tabular}{lcc}
\multicolumn{1}{c}{ Variável } & $\begin{array}{c}\text { Frequência } \\
\text { absoluta }\end{array}$ & \% \\
\hline Tempo (minutos) & 79 & $50,0 \%$ \\
01,00 a 03,63 & 35 & $22,2 \%$ \\
03,64 a 06,25 & 21 & $13,3 \%$ \\
06,26 a 08,87 & 14 & $08,9 \%$ \\
08,88 a 11,49 & 9 & $05,6 \%$ \\
11,50 a 14,11 & & \\
\hline Tempo (anos/download) & 91 & $57,6 \%$ \\
\hline 01,00 a 02,69 & 50 & $31,6 \%$ \\
02,70 a 05,19 & 9 & $5,7 \%$ \\
05,20 a 07,69 & 7 & $4,5 \%$ \\
07,70 a 10,19 & 1 & $0,6 \%$ \\
10,20 a 12,69 & &
\end{tabular}

Número de visualizações

$\begin{array}{lll}0.000 \text { a } 2.000 & 40 & 25,3 \% \\ 2.001 \text { a } 6.000 & 37 & 23,4 \% \\ 6.001 \text { a } 18.000 & 39 & 24,7 \% \\ 18.001 \text { a } 50.000 & 22 & 13,9 \% \\ >50.000 & 20 & 12,7 \%\end{array}$

\section{Identidade da conta}

Profissional da área da saúde

$107 \quad 67,7 \%$

Profissionais de outra área

$12 \quad 7,6 \%$

Ambos os profissionais

$18 \quad 11,4 \%$

Utilizador independente

$21 \quad 13,3 \%$

Sociedades Públicas

$0 \%$

\section{Aprovações (likes)}

0 a 50

$39 \quad 24,7 \%$

51 a 200

$45 \quad 28,5 \%$

201 a 500

$34 \quad 21,5 \%$

501 a 1.400

$23 \quad 14,6 \%$

$>1.400$

$17 \quad 10,7 \%$

\section{Reprovações (dislikes)}

$\begin{array}{lll}0 \text { a } 1 & 60 & 38,0 \% \\ 2 \text { a } 3 & 34 & 21,5 \% \\ 4 \text { a } 12 & 30 & 19,0 \% \\ 13 \text { a } 40 & 19 & 12,0 \% \\ >40 & 15 & 9,5 \% \\ \text { Comentários } & & \\ 00 \text { a } 01 & 64 & 40,5 \% \\ 02 \text { a } 09 & 10 & 06,3 \% \\ 10 \text { a } 25 & 23 & 14,6 \% \\ 26 \text { a } 100 & 25 & 15,8 \% \\ >100 & 36 & 36,8 \%\end{array}$


(conclusão)

\begin{tabular}{lcc}
\multicolumn{1}{c}{ Variável } & $\begin{array}{c}\text { Frequência } \\
\text { absoluta }\end{array}$ & $\%$ \\
Objetivo & 72 & $45,6 \%$ \\
Informação ao leigo & 43 & $27,2 \%$ \\
Capacitação profissional & 38 & $24,1 \%$ \\
Compartilhar experiências & 3 & $1,8 \%$ \\
Discutir terapias alternativas & 2 & $1,3 \%$ \\
Outros & & \\
\hline
\end{tabular}

Fonte: elaboração dos autores.

A Tabela 2 apresenta a distribuição de frequências dos dados relativos à acurácia dos vídeos quanto às informações sobre os temas discutidos. A maioria dos vídeos $(91,8 \%)$ não apresentou a definição do que é o câncer de mama. Quanto às informações de cunho epidemiológico, 10,4\% dos vídeos debateram esse aspecto, e 1,6\% continham informações totalmente certas. Sobre fatores de risco, 13,5\% dos vídeos informaram sobre o tópico, mas apenas 4,1\% forneceram informações totalmente certas. Para sinais e sintomas, $15,4 \%$ abordaram o tópico e 3,6\% de forma totalmente certa. A maioria dos vídeos $(85,4 \%)$ não abordou conteúdo sobre prevenção primária e dos que abordaram apenas 2,2\% continham informações totalmente corretas. A prevenção secundária foi abordada pela maioria dos vídeos (71,5\%), mas apenas $1,9 \%$ deles com informações totalmente corretas. Quanto à prevenção terciária, a maioria $(63,9 \%)$ não apresentou informações sobre o tópico, e dos que apresentaram apenas 3,8\% estavam corretos.

Tabela 2 - Distribuição de frequências dos dados relativos à acurácia dos vídeos quanto às informações abordadas sobre os temas discutidos

\begin{tabular}{lccccc}
\multicolumn{1}{c}{ Tópico } & $\begin{array}{c}\text { Totalmente } \\
\text { errado }\end{array}$ & $\begin{array}{c}\text { Parcialmente } \\
\text { errado }\end{array}$ & Não abordou & $\begin{array}{c}\text { Parcialmente } \\
\text { certa }\end{array}$ & $\begin{array}{c}\text { Totalmente } \\
\text { certa }\end{array}$ \\
Definição & $01(0,3 \%)$ & $02(0,6 \%)$ & $290(91,8 \%)$ & $9(2,8 \%)$ & $14(4,4 \%)$ \\
Epidemiologia & $03(0,6 \%)$ & $05(1,6 \%)$ & $270(85,4 \%)$ & $33(10,4 \%)$ & $02(1,6 \%)$ \\
Fatores de risco & $01(0,3 \%)$ & $03(0,9 \%)$ & $273(86,4 \%)$ & $26(8,2 \%)$ & $13(4,1 \%)$ \\
Sinais e sintomas & $00(0,0 \%)$ & $01(0,3 \%)$ & $267(84,5 \%)$ & $34(10,8 \%)$ & $14(3,6 \%)$ \\
Prevenção primária & $00(0,0 \%)$ & $03(0,9 \%)$ & $270(85,4 \%)$ & $36(11,4 \%)$ & $07(2,2 \%)$ \\
Prevenção & $10(3,2 \%)$ & $37(11,7 \%)$ & $226(71,5 \%)$ & $37(11,7 \%)$ & $06(1,9 \%)$ \\
secundária & $10(3,2 \%)$ & $16(5,1 \%)$ & $202(63,9 \%)$ & $76(24,1 \%)$ & $12(3,8 \%)$ \\
Prevenção terciária & $16 \%)$ & & &
\end{tabular}

Fonte: elaboração dos autores.

Os dados da distribuição de frequências dos dados relativos à acurácia das informações contidas nos vídeos estão apresentados na Tabela 3. A quantidade de respostas sem abrangência nos tópicos sobre câncer de mama foi $81,31 \%$ e somente $14,50 \%$ das respostas estavam certas ou parcialmente certas. 
Tabela 3 - Distribuição de frequências dos dados relativos à acurácia das informações contidas nos vídeos

\begin{tabular}{lcc} 
Acurácia das informações & $\begin{array}{c}\text { Frequência } \\
\text { absoluta }\end{array}$ & $\begin{array}{c}\text { Frequência } \\
\text { relativa }\end{array}$ \\
\hline Totalmente erradas & 25 & $1,14 \%$ \\
Parcialmente erradas & 67 & $3,05 \%$ \\
Não abrange os tópicos & 1789 & $81,31 \%$ \\
Parcialmente certas & 251 & $11,41 \%$ \\
Totalmente certas & 68 & $3,09 \%$ \\
Total & $\mathbf{2 2 0 0}$ & $\mathbf{1 0 0 \%}$ \\
\hline
\end{tabular}

Fonte: elaboração dos autores.

A Tabela 4 apresenta os dados da distribuição de frequências da qualidade dos vídeos baseada na acurácia das informações sobre os tópicos relacionados ao câncer de mama, oriundo da pontuação obtida no instrumento de análise. Mais de $53 \%$ dos vídeos foram avaliados como ruins ou péssimos e apenas 6,2\% deles avaliados como bons ou ótimos.

Tabela 4 - Distribuição de frequências da qualidade dos vídeos baseada na acurácia das informações

\begin{tabular}{lcc} 
Tipo de conteúdo & $\begin{array}{c}\text { Frequência } \\
\text { absoluta }\end{array}$ & $\begin{array}{c}\text { Frequência } \\
\text { relativa }\end{array}$ \\
\hline Péssimo & 49 & $31,0 \%$ \\
Ruim & 36 & $22,8 \%$ \\
Regular & 63 & $40,0 \%$ \\
Bom & 7 & $4,4 \%$ \\
Ótimo & 3 & $1,8 \%$ \\
Total & $\mathbf{1 5 8}$ & $\mathbf{1 0 0 \%}$ \\
\hline
\end{tabular}

Fonte: elaboração dos autores.

\section{DISCUSSÃO}

Diante das evidências de que a plataforma de vídeos online YouTube é frequentemente utilizada pelas pessoas para acessar conteúdo informativo sobre saúde na Web (CARVALHO; GASTALDO, 2019; ALVES; ALVES; TEIXEIRA, 2019; PALMEIRA et al., 2019; RODRIGUES et al., 2018) e de que muito dos conteúdos postados apresentam baixa qualidade (CÓRDULA; ARAÚJO; SILVA, 2019; ODDONE; FRANÇA et al., 2019), buscou-se, no presente estudo, compreender como o YouTube disponibiliza conteúdo sobre o câncer de mama aos brasileiros, bem como avaliar a qualidade dessas informações. De modo geral, os dados aqui mostrados apontam que muitos dos vídeos apresentam conteúdo de baixa qualidade, embora tenham sido postados por contas autodeclaradas como de profissionais da área da saúde, e que, mesmo com tantas limitações, eles tiveram grande audiência e foram amplamente apreciados.

Esse achado é preocupante, visto que uma das principais barreiras para a adesão das mulheres aos programas de controle é o seu conhecimento limitado sobre o processo saúde-doença (LABARGE; BROOM, 2019; YAMAGUCHI et al., 2020). Como as chances de cura do câncer de mama dependem muito da precocidade do diagnóstico (INCA, 2020; BERNARDES et al., 2019; SOUSA, 2020), qualquer fator que interfira no gerenciamento do autocuidado da mulher pode ser decisivo para o desfecho de sua saúde e 
qualidade de vida. Os motivos que fazem, ou fizeram, as pessoas assistirem aos vídeos e os compartilharem não foram aqui investigados, mas independentemente disso, o que chama a atenção é a popularidade, tendo alguns deles atingindo mais de 50 mil visualizações. O público-alvo, normalmente mulheres, reconhecidamente, é bastante ativo nas redes sociais, especialmente na busca de informações sobre saúde (BRACHTENBACH et al., 2020).

Outro fator importante diz respeito a algumas características de edição dos vídeos, como duração e objetivo. Embora não se tenha avaliado a correlação desses fatores com a popularidade, alguns estudos têm mostrado que os vídeos online de curta duração são os mais vistos (O'REILLY, 2019; UKOHA; STRANIERI, 2019) e aqueles cujo objetivo é esclarecimento de dúvidas sobre saúde ou compartilhamento de experiências são os que mais têm atraído os internautas (UKOHA; STRANIERI, 2019). De fato, a maioria dos vídeos aqui analisados são curtos, em sua maioria com menos de seis minutos, e direcionados especialmente para esclarecimentos ao leigo e trocas de experiências.

Não se pode também descartar a influência da identidade da conta que postou o vídeo. Como a maioria deles é oriunda de contas daqueles que se autodeclararam ser profissionais da área da saúde, isso pode ter gerado sensação de confiança e amplificado o intuito de visualizar. Estudos que avaliaram interatividade nas postagens do YouTube, sobre saúde, mostram que a presença de um profissional dessa área no vídeo ou na própria identidade da conta é determinante para sua popularidade (YURDAISIK, 2020).

Obviamente, uma limitação importante de nossos dados é a heterogeneidade no tempo de exposição dos vídeos, a qual influencia a contabilização do número de visualizações. Como essa questão não foi considerada na análise, não se pode descartar a influência desse efeito; no entanto, como a média de visualizações foi cerca de 16 mil por vídeo, e a média de exposição foi cerca de dois anos, é possível concluir que uma grande parcela dos vídeos obteve, pelo menos, 8 mil visualizações por ano.

Independentemente do motivo, o que se mostra aqui é que há audiência para vídeos sobre o câncer de mama no YouTube, e isso não pode ser ignorado pelos gestores de saúde pública. Por outro lado, mesmo diante de tamanha popularidade, as ações de interação social permitidas na plataforma, como curtidas e comentários foram muito pouco exploradas pelas pessoas que visualizaram os vídeos. A maioria deles recebeu menos de 200 ações do tipo like e apenas quatro do tipo dislike, e o número de comentários não passou a marca dos 40. Essa indiferença interativa sugere um comportamento apático dos internautas, que enxergam a plataforma apenas como uma fonte fácil e rápida de conteúdo informativo, sem aproveitar seu potencial de rede de sociabilização.

Esse fato gera certa preocupação, pois um dos pilares do processo de aprendizado em rede é a troca de ideias, exposição de dúvidas e questionamentos (VAN DER VAART; DROSSAERT, 2017). E o que tem feito das RSO tão importantes para o aprendizado em saúde é exatamente o uso do seu potencial interativo, de construção conjunta de ideias, fundamental para o aprendizado. Vários estudos têm mostrado quão benéfico é o uso das RSO para construção do conhecimento em saúde, tanto individual, quanto coletivo (BASCH et al., 2015).

Outro ponto importante aqui apresentado é sobre o conteúdo dos vídeos. Como mostrado, mais de 90\% deles não se ocuparam de dar uma definição sobre o que é o câncer de mama. Pensando na contribuição do conhecimento sobre o processo saúde-doença-cuidado para o controle do câncer de mama (FERREIRA; PIAZZA; SOUZA et al., 2019), a ausência de uma definição clara sobre a doença pode limitar a compreensão subsequente de outros tópicos mais específicos do processo, como a discussão de sinais e sintomas, e ainda dos fatores de risco, por exemplo. Menos de $15 \%$ dos vídeos abordaram informações sobre os sinais e sintomas, e quando o fizeram, grande parte das informações era equivocada.

Cenário parecido ocorreu no que diz respeito aos fatores de risco, pois menos de $14 \%$ dos vídeos abordaram este tema, e apenas $4 \%$ do total dos vídeos o fizeram de forma correta. Do ponto de vista da 
saúde pública, a compreensão da mulher quanto aos principais sinais e sintomas do câncer de mama é fundamental para o sucesso das ações de rastreio e diagnóstico precoce desta malignidade (KOLAK et al., 2017). Embora o autoexame das mamas não seja completamente indicado para as mulheres como um método para diagnóstico (INCA, 2020; HUSNA et al., 2019), a identificação de qualquer sinal sugestivo de malignidade é propulsionadora de um movimento de procura do serviço de saúde (MIGOWSKI et al., 2018), e isso facilita que a mulher adentre no sistema de saúde e acesse os serviços de diagnóstico precoce.

A compreensão correta dos fatores de risco para o câncer de mama é decisiva para o desenvolvimento e a propagação de comportamentos protetivos a esse tipo de câncer (MIGOWSKI et al., 2018) e fundamental para o controle populacional desse tipo de câncer, especialmente em um país tão desigual quanto o Brasil, em termos de oferta de serviços de saúde de qualidade, onde os dados epidemiológicos para esse tipo de câncer têm apontado diferenças regionais importantes (FERREIRA et al., 2019). Não apenas a omissão de informações sobre esses aspectos do câncer de mama, mas a propagação de informações incorretas sobre o tema configuram um grande desserviço ao sistema de saúde pública, e colocam em risco a viabilidade do uso do YouTube como ferramenta coadjuvante no processo de educação em saúde da população.

Conteúdos informativos mais pragmáticos de controle do câncer de mama, como aqueles referentes à prevenção primária, secundária e terciária, também foram objeto temático dos vídeos no YouTube. Infelizmente, como o esperado, já que não há uma tendência dos youtubers a produzirem e postarem vídeos sobre fatores de risco e de proteção, nem tampouco mostram uma preferência por essas questões, o tema da prevenção primária foi explorado em apenas $15 \%$ dos vídeos, e apenas $3 \%$ deles o expuseram de forma correta. Esse achado é alarmante, pois em termos de gerenciamento das ações de controle do câncer de mama, especialmente em países em desenvolvimento, como o Brasil, as ações de prevenção primária são decisivas para o controle da malignidade (INCA, 2020).

A ausência de conhecimento sobre o tema, ou ainda pior, a construção de um conhecimento equivocado sobre o assunto pode fazer com que muitas mulheres desenvolvam e propaguem ideias distorcidas sobre como se prevenir do câncer de mama. Mais uma vez, diante das grandes desigualdades regionais no controle do câncer no Brasil (OHL et al., 2016), a adesão da população às estratégias preventivas pode ser decisiva para o desfecho de saúde. Por outro lado, na maioria dos vídeos, mais de $70 \%$, foram vinculados conteúdos sobre prevenção secundária. Esse fato é relevante, no sentido de que qualquer iniciativa de melhorar a adesão das mulheres aos programas de rastreio e detecção precoce tem um valor imensurável para a saúde pública, especialmente na medida em que as taxas de sobrevida aumentam consideravelmente quando o diagnóstico é precoce, como é o caso do câncer de mama (BERNARDES et al., 2019; SOUSA, 2020).

Muitos estudos têm mostrado que a limitação de conhecimento sobre a prevenção secundária configura uma forte barreira para adesão das mulheres ao rastreio do diagnóstico do câncer de mama (HERR et al., 2013), e, por esse motivo, o grande volume de vídeos sobre o tema tende a contribuir com esse aspecto frágil do sistema de controle desse tipo de câncer. No entanto, o que se foi aqui constatado é que menos de $2 \%$ dos vídeos analisados continham informações sobre prevenção secundária de forma correta. Mais uma vez, isso reforça a ideia de que, até o momento, os vídeos disponibilizados no Youtube, para as mulheres brasileiras, pouco contribuem, em termos de educação em saúde, para a melhoria do controle desse tipo de câncer.

A ausência de vídeos com conteúdo informativo sobre prevenção terciária, ignora, também, os internautas que, porventura, buscam informações para si próprios ou para um conhecido com diagnóstico de câncer de mama, prejudicando, também, a construção de um conhecimento mais amplo sobre todos os aspectos da doença. De forma geral, como aqui apresentado, a maioria dos vídeos foram avaliados como ruins, em termos de qualidade do conteúdo, o que revela a necessidade imediata de maiores discussões sobre o papel das RSO no contexto da saúde pública, em especial da plataforma de vídeos YouTube. 
Reciis - Revista Eletrônica de Comunicação, Informação \& Inovação em Saúde, Rio de Janeiro, v. 15, n. 3, p. 648-664, jul.-set. 2021 [www.reciis.icict.fiocruz.br] e-ISSN 1981-6278

\section{CONCLUSÃO}

Conclui-se que os vídeos sobre o câncer de mama disponíveis na plataforma YouTube para os brasileiros apresentam conteúdo de qualidade limitada. Esta evidência sugere que o uso desses vídeos como fonte de informações em saúde pouco contribui para o incentivo de comportamentos de autocuidado necessários para a prevenção do câncer de mama uma vez que o direcionamento dos temas abordados se desvincula do proposto pelos órgãos regulamentadores de saúde pública.

\section{REFERÊNCIAS}

ALBARADO, Ádria Jane et al. Análise da recepção de campanhas audiovisuais de saúde no Brasil: um estudo qualitativo sobre a percepção da população no controle do Aedes. In: CONGRESSO IBERO-AMERICANO EM INVESTIGAÇÃO QUALITATIVA, 8., 2019, Lisboa. Atas CIAIQ2019, Aveiro, v. 2, p. 1343-1354, 2019. Disponível em: https://proceedings.ciaiq.org/index.php/CIAIQ2019/article/view/2292. Acesso em: 9 jul. 2021.

ALVES, Natália Christina de Moura; ALVES, Martha Lorena de Moura; TEIXEIRA, Flaviana Tavares Vieira. Empoderamento: o caminho para alcançar a igualdade de gênero. Raízes e Rumos, Rio de Janeiro, v. 6, n. 2, p. 111-126, 2019. Disponível em: http://www.seer.unirio.br/raizeserumos/article/view/8271. Acesso em: 21 jul. 2021.

AMORIM, Vivian Mae Schmidt Lima et al. Fatores associados à não realização da mamografia e do exame clínico das mamas: um estudo de base populacional em Campinas, São Paulo, Brasil. Cadernos de Saúde Pública, Rio de Janeiro, v. 24, n. 11, p. 2623-2632, 2008. DOI: https://doi.org/10.1590/S0102311X2008001100017. Disponível em: https://www.scielo.br/j/csp/a/H4DVcpsh47gff8cnxPvYLZn/?lang=pt. Acesso em: 6 jul. 2021.

BACKINGER, Cathy L. et al. YouTube as a source of quitting smoking information. Tobacco Control, [s. I.], v. 20, n. 2, p. 119-122, 2011. DOI: http://dx.doi.org/10.1136/tc.2009.035550. Disponível em: https:// tobaccocontrol.bmi.com/content/20/2/119. Acesso em: 6 jul. 2021.

BASCH, Corey H. et al. Characteristics of YouTube ${ }^{\mathrm{TM}}$ videos related to mammography. Journal of Cancer Education, [s. I.], v. 30, n. 4, p. 699-703, 2015. DOI: https://doi.org/10.1007/s13187-014-0769-9. Disponível em: https://link.springer.com/article/10.1007/s13187-014-0769-9. Acesso em: 6 jul. 2021.

BENEDICTIS, Anna de et al. WhatsApp in hospital? An empirical investigation of individual and organizational determinants to use. PloS One, [s. I.], v. 14, n. 1, p. e0209873, 2019. DOI: https://doi.org/10.1371/journal. pone.0209873. Disponível em: https://journals.plos.org/plosone/article?id=10.1371/journal.pone.0209873. Acesso em: 6 jul. 2021.

BERNARDES, Nicole Blanco et al. Câncer de mama x diagnóstico. Id On Line: Revista Multidisciplinar e de Psicologia, Jaboatão dos Guararapes, v. 13, n. 44, p. 877-885, 2019. DOI: https://doi.org/10.14295/idonline. v13i44.1636. Disponível em: https://idonline.emnuvens.com.br/id/article/view/1636. Acesso em: 22 jul. 2021.

BRACHTENBACH, Travis et al. YouTube: Searching for answers about breast cancer. Breast Disease, [s. I.], v. 39, n. 2, p. 85-90, 2020. DOI: https://doi.org/10.3233/BD-200445. Disponível em: https://content.iospress. com/articles/breast-disease/bd200445. Acesso em: 6 jun. 2021.

BRASIL. Ministério da Saúde. Agência Nacional de Vigilância Sanitária. Resolução - RDC n. 36, de 25 de julho de 2013. Institui ações para a segurança do paciente em serviços de saúde e dá outras providências. Diário Oficial da União, Brasília, DF, 26 jul. 2013. Disponível em: https://bvsms.saude.gov.br/bvs/saudelegis/ anvisa/2013/rdc0036_25_07_2013.html. Acesso em: 5 jul. 2021.

CARVALHO, Sérgio Resende; GASTALDO, Denise. Promoção à saúde e empoderamento: uma reflexão a partir das perspectivas crítico-social pós-estruturalista. Ciência \& Saúde Coletiva, Rio de Janeiro, v. 13, n. suppl 2, p. 2029-2040, 2008. DOI: https://doi.org/10.1590/S1413-81232008000900007. Disponível em: https:// www.scielo.br/j/csc/a/T9KWHWF5xcb3kmxTM4BLTQx/?lang=pt. Disponível em: 21 jul. 2021.

CHOU, Wen-Ying Sylvia; HUNT, Yvonne; FOLKERS, Anna; AUGUSTSON. Cancer survivorship in the age of YouTube and social media: a narrative analysis. Journal of Medical Internet Research, [s. I.], v. 13, n. 1, p. e7, 2011. DOI: https://doi.org/10.2196/jmir.1569. Disponível em: https://www.jmir.org/2011/1/e7l. Acesso em: 6 jul. 2021. 
CÓRDULA, Flavio Ribeiro; ARAÚJO, Wagner Junqueira de; SILVA, Alzira Karla Araújo da. Análise de correlação entre as IES brasileiras e a participação de sua comunidade acadêmica em redes sociais científicas. InCID: Revista de Ciência da Informação e Documentação, São Paulo, v. 10, n. 1, p. 201-218, 2019. DOI: https://doi.org/10.11606/issn.2178-2075.v10i1p201-218. Disponível em: https://www.revistas.usp.br/ incid/article/view/149259. Acesso em: 21 jul. 2021.

CURRAN, Vernon et al. A review of digital, social, and mobile technologies in health professional education. Journal of Continuing Education in the Health Professions, v. 37, n. 3, p. 195-206, 2017. DOI: http://dx.doi. org/10.1097/CEH.0000000000000168. Disponível em: https://journals.lww.com/jcehp/Abstract/2017/03730/A Review_of_Digital,_Social,_and_Mobile.8.aspx. Acesso em: 6 jul. 2021.

DAWSON, Annelise Lorelei et al. Online videos to promote sun safety: results of a contest. Dermatology Reports, [s. I.], v. 3, n. 1, p. 17-19, 2011. DOI: http://dx.doi.org/10.4081/dr.2011.e9. Disponível em: https://www. pagepress.org/journals/index.php/dr/article/view/dr.2011.e9. Acesso em: 6 jul. 2021.

DOS-SANTOS-SILVA, Isabel. Políticas de controle do câncer de mama no Brasil: quais são os próximos passos?. Cadernos de Saúde Pública, Rio de Janeiro, v. 34, n. 6, p. e00097018, 2018. DOI: https://doi. org/10.1590/0102-311X00097018. Disponível em: https://www.scielo.br/j/csp/a/c3Ymmf3gpWZW5xqHnQLJLW c/?lang=pt. Acesso em: 5 jul. 2021.

EYSENBACH, Gunther; KÖHLER, Christian. How do consumers search for and appraise health information on the world wide web? Qualitative study using focus groups, usability tests, and in-depth interviews. BMJ, [s. I.], v. 324, p. 573-577, 2002. DOI: https://doi.org/10.1136/bmj.324.7337.573. Disponível em: https://www.bmj.com/ content/324/7337/573. Acesso em: 5 jul. 2021.

FERREIRA, Daiane Medeiros Roque; LIMA, Afonso Augusto Teixeira de Freitas de Carvalho; FERREIRA, Marco Aurélio Marques. Dimensões estatal, gerencial e individual da Política Nacional de Prevenção e Controle do Câncer (de Mama): um modelo avaliativo. Administração Pública e Gestão Social, Viçosa, MG, v. 11, n. 4, p. 1-19, 2019. Disponível em: https://periodicos.ufv.br/apgs/article/view/7240. Acesso em: 22 jul. 2021.

FERREIRA, lago Gonçalves; PIAZZA, Marina; SOUZA, Deyse. Oficina de saúde e sexualidade: residentes de saúde promovendo educação sexual entre adolescentes de escola pública. Revista Brasileira de Medicina de Família e Comunidade, Rio de Janeiro, v. 14, n. 41, p. 1788, 2019. DOI: https://doi.org/10.5712/ rbmfc14(41)1788. Disponível em: https://rbmfc.org.br/rbmfc/article/view/1788. Acesso em: 22 jul. 2021.

FIGUEIREDO, Francisco Winter dos Santos et al. The role of health policy in the burden of breast cancer in Brazil. BMC Women's Health, [s. I.], v. 17, n. 1, p. 1-6, 2017. DOI: https://doi.org/10.1186/s12905-017-0477-9. Disponível em: https://bmcwomenshealth.biomedcentral.com/articles/10.1186/s12905-017-0477-9. Acesso em: 6 jul. 2021.

FRANÇA, Tania; RABELLO, Elaine Teixeira; MAGNAGO Carinne. As mídias e as plataformas digitais no campo da Educação Permanente em Saúde: debates e propostas. Saúde em Debate, Rio de Janeiro, v. 43, p. 106-115, 2019. DOI: https://doi.org/10.1590/0103-11042019s109. Disponível em: https://www.scielo.br/j/sdeb/a/ GsRWdhS9VztCddQjNT46RkN/?lang=pt. Acesso em: 6 jul. 2021.

FROSSARD, Vera Cecília; DIAS, Maria Clara Marques. O impacto da internet na interação entre pacientes: novos cenários em saúde. Interface - Comunicação, Saúde, Educação, Botucatu, v. 20, n. 57, p. 349-361, 2016. DOI: https://doi.org/10.1590/1807-57622014.1334. Disponível em: https://www.scielo.br/j/icse/a/Xj5Hwb9 FQG3G6D8xDWZ3XWJ/?lang=pt. Acesso em: 6 jul. 2021.

FROTA, Natasha Marques et al. Construction of an educational technology for teaching about nursing on peripheral venipuncture. Revista Gaúcha de Enfermagem, Porto Alegre, v. 34, n. 2, p. 29-36, 2013. DOI: http://dx.doi.org/10.1590/S1983-14472013000200004. Disponível em: https://www.scielo.br/j/rgenf/a/q4JDW55 WxNXtX3g3nVNgscg/?lang=en. Acesso em: 6 jul. 2021.

GLOBAL CANCER OBSERVATORY (GLOBOCAN). Cervix uteri. March, 2020. p.2. Disponível em: https://gco. iarc.fr/today/data/factsheets/cancers/23-Cervix-uteri-fact-sheet.pdf. Acesso em: 22 jan. 2020.

HERR, Gerli Elenise et al. Avaliação de conhecimentos acerca da doença oncológica e práticas de cuidado com a saúde. Revista Brasileira de Cancerologia, Rio de Janeiro, v. 59, n. 1, p. 33-41, 2013. DOI: https://doi. org/10.32635/2176-9745.RBC.2013v59n1.540. Disponível em: https://rbc.inca.gov.br/revista/index.php/revistal article/view/540. Acesso em: 22 jul. 2021. 
HILLYER, Grace Clarke et al. YouTube videos as a source of information about mastectomy. The Breast Journal, [s. I.], v. 25, n. 2, p. 349-350, 2019. DOI: https://doi.org/10.1111/tbj.13222. Disponível em: https:// onlinelibrary.wiley.com/doi/10.1111/tbj.13222. Acesso em: 6 jun. 2021.

HUSNA, Putri Halimu et al. Breast self-examination education for skill and behavior. Education Health, Abingdon, v. 32, n. 2, p. 101-102, 2019. Disponível em: https://pubmed.ncbi.nlm.nih.gov/31745006/. Acesso em: 22 jul. 2021.

INSTITUTO NACIONAL DE CÂNCER (INCA). Estimativa 2012: incidência de câncer no Brasil. Rio de Janeiro: Inca, 2020.

KELLY, Brendan S. et al. The use of Twitter by radiology journals: an analysis of Twitter activity and impact factor. Journal of the American College of Radiology, [s. I.], v. 13, n. 11, p. 1391-1396, 2016. DOI: https:// doi.org/10.1016/j.jacr.2016.06.041. Disponível em: https://www.jacr.org/article/S1546-1440(16)30557-9/fulltext. Acesso em: 6 jun. 2021.

KOLAK, Agnieszka et al. Primary and secondary prevention of breast cancer. Annals of Agricultural and Environmental Medicine, Lublin, v. 24, n. 4, p. 549-553, 2017. DOI: https://doi.org/10.26444/aaem/75943. Disponível em: http://www.aaem.pl/Primary-and-secondary-prevention-of-breast-cancer,75943,0,2.html. Acesso em: 22 jul. 2021

LABARGE, Gene; BROOM, Matt. Social media in Primary Care. Missouri Medicine, Jefferson City, MO, v. 116, n. 2, p. 106-110, 2019. Disponível em: https://www.ncbi.nlm.nih.gov/pmc/articles/PMC6461310/. Acesso em: 21 jul. 2021.

LANGFORD, Aisha; LOEB, Stacy. Perceived patient-provider communication quality and sociodemographic factors associated with watching health-related videos on YouTube: a cross-sectional analysis. Journal of Medical Internet Research, [s. I.], v. 21, n. 5, p. e13512, 2019. DOI: https://doi.org/10.2196/13512. Disponível em: https://www.jmir.org/2019/5/e13512/. Acesso em: 6 jun. 2021.

LIMA, Vanessa Faria de; MAZZA, Verônica de Azevedo; SCOCHI, Carmen Gracinda Silvan; GONÇALVES, Luciana Schleder. Uso de informações on-line sobre saúde/doença por famílias de prematuros hospitalizados. Revista Brasileira de Enfermagem, Brasília, DF, v. 72, n. 3, p. 79-87, 2019. DOI: http://dx.doi. org/10.1590/0034-7167-2018-0030. Disponível em: https://www.scielo.br/j/reben/a/L99b6CtqtfWBfLnMtyd4yTf l?lang=pt. Acesso em: 9 jun. 2021.

MIGOWSKI, Arn et al. Diretrizes para detecção precoce do câncer de mama no Brasil. Il-Novas recomendações nacionais, principais evidências e controvérsias. Cadernos de Saúde Pública, Rio de Janeiro, v. 34, p. e00074817, 2018. DOI: https://doi.org/10.1590/0102-311X00074817. Disponível em: https:// www.scielo.br/j/csp/a/8gGyb5s9Nt3nSsw5GFnnPQb/?lang=pt. Acesso em: 6 jun. 2021.

MORETZSOHN, Sylvia Debossan. Uma legião de imbecis: hiperinformação, alienação e o fetichismo da tecnologia libertária. Liinc em Revista, Rio de Janeiro, v. 13, n. 2, p. 294-306, 2017. DOI: https://doi. org/10.18617/liinc.v13i2.4088. Disponível em: http://revista.ibict.br/liinc/article/view/4088. Acesso em: 6 jun. 2021.

MOTA, João de Jesus Oliveira et al. Análise de conteúdos de posts sobre alimentação divulgados por influenciadoras digitais na rede social Instagram. DEMETRA: Alimentação, Nutrição \& Saúde, Rio de Janeiro, v. 14, p. e39076, 2019. Disponível em: https://www.e-publicacoes.uerj.br/index.php/demetra/article/ view/39076. Acesso em: 6 jun. 2021.

O'REILLY, Michelle et al. Potential of social media in promoting mental health in adolescents. Health Promotion International, [s. I.], v. 34, n. 5, p. 981-991, 2019. DOI: https://doi.org/10.1093/heapro/day056. Disponível em: https://academic.oup.com/heapro/article-abstract/34/5/981/5061526?redirectedFrom=fulltext. Acesso em: 22 jul. 2021.

ODDONE, Nanci Elizabeth; FRANÇA, Cláudio Márcio de. Plataformas de livros acadêmicos em acesso aberto e sua representação no Twitter: métricas de divulgação, descoberta e avaliação. Transinformação, Campinas, v. 31, n. 1, p. e190011, 2019. DOI: http://dx.doi.org/10.1590/2318-0889201931e190011. Disponível em: https://www.scielo.br/j/tinf/a/CLgcratKYN4Jd46wBP6w75D/?lang=pt. Acesso em: 21 jul. 2021.

OHL, Isabella Cristina Barduchi et al. Ações públicas para o controle do câncer de mama no Brasil: revisão integrativa. Revista Brasileira de Enfermagem, Brasília, DF, v. 69, n. 4, p. 793-803, 2016. DOI: http://dx.doi. org/10.1590/0034-7167.2016690424i. Disponível em: https://www.scielo.br/j/reben/a/6TL9tKq7vNXvkQRMsWr nyNv/?lang=pt. Acesso em: 22 jul. 2021. 
Reciis - Revista Eletrônica de Comunicação, Informação \& Inovação em Saúde, Rio de Janeiro, v. 15, n. 3, p. 648-664, jul.-set. 2021 [www.reciis.icict.fiocruz.br] e-ISSN 1981-6278

ORGANIZAÇÃO PAN-AMERICANA DA SAÚDE (OPAS). Câncer. Brasília, DF: Opas, 2020. Disponível em: https://www.paho.org/pt/topicos/cancerl. Acesso em: 5 jun. 2021.

PALMEIRA, lací Proença et al. Devolutiva de resultados de pesquisa a profissionais de saúde no contexto HIV. Revista Brasileira de Enfermagem, Brasília, DF, v. 72, n. 5, p. 1416-1420, 2019. DOI: http://dx.doi. org/10.1590/0034-7167-2017-0875. Disponível em: https://www.scielo.br/j/reben/a/NL9St8ZqDXxfBDKVjR8gC Xn/?lang=pt. Acesso em: 21 jul. 2021.

PANDER, Tanja; PINILLA, Severin; DIMITRIADIS, Konstantinos; FISCHER, Martin R. The use of Facebook in medical education - a literature review. GMS Journal for Medical Education, Erlangen, v. 31, n. 3, p. 1-19, 2014. DOI: https://doi.org/10.3205/zma000925. Disponível em: https://www.egms.de/static/en/journals/ zma/2014-31/zma000925.shtml. Acesso em: 6 jul. 2021.

PINHEIRO, Cleoneide Paulo Oliveira et al. Procrastinação na deteç̧ão precoce do câncer de mama. Revista Brasileira de Enfermagem, Brasília, DF, v. 72, n. 3, p. 227-234, 2019. DOI: https://doi.org/10.1590/0034-71672018-0547. Disponível em: https://www.scielo.br/j/reben/a/bfvs6GWYgfGwBN6RW7zr7vL/?lang=pt. Acesso em: 6 jul. 2021.

RODRIGUES, Andreia Sias et al. Um estudo de Mapeamento Sistemático sobre metodologias de avaliação em Interação Humano-Computador voltadas à Tecnologia Assistiva com foco em Pessoas com Deficiência Motora. iSys - Brazilian Journal of Information Systems, Porto Alegre, v. 11, n. 3, p. 90-126, 2018. DOI: https://doi.org/10.5753/isys.2018.372. Disponível em: https://sol.sbc.org.br/journals/index.php/isys/article/ view/372. Acesso em: 21 jul. 2021.

SADEGHI, Roya; HESHMATI, Hashem. Innovative methods in teaching college health education course: a systematic review. Journal of Education and Health Promotion, Isfahan, v. 8, n. 1, p. 1-8, 2019. Disponível em: https://www.jehp.net/article. asp?issn=2277-9531; year=2019; volume=8;issue=1; spage=103; epage=103; aul ast=Sadeghi. Acesso em: 6 jul. 2021.

SALVADOR, Pétala Tuani Candido de Oliveira et al. Segurança do paciente: caracterização de vídeos do Youtube. Revista Gaúcha de Enfermagem, Porto Alegre, v. 38, n. 1, p. e61713, 2017. DOI: https://doi. org/10.1590/1983-1447.2017.01.61713. Disponível em: https://www.scielo.brlj/rgenf/a/RQfXL3VpFLb8PRpsvZ 8Hfrs/?lang=pt. Acesso em: 6 jul. 2021.

SELVI, Onur et al. YouTube como fonte informativa sobre bloqueios do plexo braquial: avaliação de conteúdo e valor educativo. Brazilian Journal of Anesthesiology, Rio de Janeiro, v. 69, n. 2, p. 168-176, 2019. DOI: https://doi.org/10.1016/j.bjane.2018.12.005. Disponível em: https://www.sciencedirect.com/science/article/pii/ S0104001418301647?via\%3Dihub. Acesso em: 6 jul. 2021.

SILVA, Francisco Vieira da; SILVA JÚNIOR, Joseeldo da. Mentiras sinceras (não) me interessam: estratégias biopolíticas do Ministério da Saúde no combate às fake news. Revista Intersecções, Jundiaí, v. 12, n. 27, p. 226-246, 2019. Disponível em: https://revistas.anchieta.br/index.php/Revistalnterseccoes/article/view/1395. Acesso em: 6 jul. 2021.

SOUSA, Citrya Jakellinne Alves. Câncer de mama em paciente do sexo masculino: a importância da atenção primária no diagnóstico e tratamento: um relato de caso. Revista Artigos.Com, São Paulo, v. 14, p. e2962, 2020. Disponível em: https://acervomais.com.br/index.php/artigos/article/view/2962. Acesso em: 22 jul. 2021.

SOUZA, Cyntia Franciele Leite; FERREIRA, Jéssica Miranda; PEREIRA; Andresa Costa; SILVA, Marco Antônio Dias da. Entendendo o uso de vídeos como ferramenta complementar de ensino. Journal of Health Informatics, [s. I.], v. 11, n. 1, p. 3-7, 2019. Disponível em: http://www.jhi-sbis.saude.ws/ojs-jhi/index.php/jhisbis/article/view/600. Acesso em: 6 jul. 2021.

SYED-ABDUL, Shabbir et al. Misleading health-related information promoted through video-based social media: anorexia on YouTube. Journal of Medical Internet Research, [s. I.], v. 15, n. 2, p. e30, 2013. DOI: https://doi.org/10.2196/imir.2237. Disponível em: https://www.pagepress.org/journals/index.php/dr/article/view/ dr.2011.e9. Acesso em: 6 jul. 2021.

TENGLAND, Per-Anders. Behavior change or empowerment: on the ethics of health-promotion strategies. Health Care Analysis, [s. I.], v. 5, n. 2, p. 140-153, 2012. DOI: https://doi.org/10.1007/s10728-013-0265-0. Disponível em: https://link.springer.com/article/10.1007/s10728-013-0265-0. Acesso em: 6 jul. 2021.

UKOHA, Chukwuma; STRANIERI, Andrew. Criteria to measure social media value in health care settings: narrative literature review. Journal of Medical Internet Research, [s. I.], v. 21, n. 12, p. e14684, 2019. DOI: https://doi.org/10.2196/14684. Disponível em: https://www.jmir.org/2019/12/e14684/. Acesso em: 22 jul. 2021. 
VALE, Paulo Roberto Lima Falcão do et al. Estratégias de empoderamento em saúde: diálogo entre o Plano Municipal e o relatório da Conferência de Saúde. Revista Gestão \& Saúde, Brasília, DF, v. 9, n. 1, p. 78-94, 2018. Disponível em: https://periodicos.unb.br/index.php/rgs/article/view/10431. Acesso em: 6 jul. 2021.

VAN DER VAART, Rosalie; DROSSAERT, Constance. Development of the digital health literacy instrument: measuring a broad spectrum of health 1.0 and health 2.0 skills. Journal of Medical Internet Research, [s. I.], v. 19, n. 1, p. e27, 2017. DOI: https://doi.org/10.2196/jmir.6709. Disponível em: https://www.jmir.org/2017/1/ e27l. Acesso em: 22 jul. 2021.

WORLD HEALTH ORGANIZATION (WHO). Global action plan for the prevention and control of noncommunicable diseases 2013-2020. Geneva: A Organização, 2013. Disponível em: https://apps.who.int/ iris/bitstream/handle/10665/94384/9789241506236 eng.pdf;jsessionid=04DDB46DD5858F0F32693804D525F 807? sequence=1/. Acesso em: 22 jan. 2020.

YAMAGUCHI, Mirian Ueda et al. O papel das mídias digitais e da literacia digital na educação nãoformal em saúde. Revista Eletrônica de Educação, São Carlos, v. 14, p. 1-11, 2020. DOI: https://doi. org/10.14244/198271993761. Disponível em: http://www.reveduc.ufscar.br/index.php/reveduc/article/ view/3761. Acesso em: 21 jul. 2021.

YURDAISIK, Isil. Analysis of the most viewed first 50 videos on YouTube about breast cancer. BioMed Research International, [s. I.], v. 2020, 2020. DOI: https://doi.org/10.1155/2020/2750148. Disponível em: https://www.hindawi.com/journals/bmri/2020/2750148/. Acesso em: 22 jul. 2021. 